\title{
ROUTING WIRELESS SENSOR NETWORKS BASED ON SOFT Computing PARAdigms: Survey
}

\author{
Marwa Sharawi ${ }^{1}$, Imane Aly Saroit ${ }^{2}$, Hesham El-Mahdy ${ }^{3}$, Eid Emary ${ }^{4}$ \\ ${ }^{1}$ Faculty of Computer Studies, Arab Open University, Egypt \\ m. sharawi@aou.edu.eg \\ ${ }^{2}$ Faculty of Computers and Information, Cairo University, Egypt \\ i.saroitefci-cu.edu.eg \\ ${ }^{3}$ Faculty of Computers and Information, Cairo University, Egypt \\ eheshamefci-cu.edu.eg \\ ${ }^{4}$ Faculty of Computers and Information, Cairo University, Egypt \\ e.emaryefci-cu.edu.eg
}

\begin{abstract}
Wireless Sensor Networks (WSNs) are defined as dynamic, self-deployed, highly constrained structured network. It's high computational environment with limited and controlled transmission range, processing, as well as limited energy sources. The sever power constraints strongly affect the existence of active nodes and hence the network lifetime. In order to prolong the network life time we have to overcome the scarcity in energy resources and preserve the processing of the sensor nodes as long as possible. Power management approaches efficiently reduce the sensor nodes energy consumption individually in each sensor node and the adaptive efficient routing technique has greatly appeals a great attention in research. The potential paradigms of soft-computing (SC) highly addressed their adaptability and compatibility to overwhelm the complex challenges in WSNs. This paper is introducing and surveying some of the Soft Computing proposed routing models for WSNs that optimally prolongs its life time.
\end{abstract}

\section{KEYWORDS}

Wireless Sensor Network, Routing, Soft Computing

\section{INTRODUCTION}

WSN is a system of distributed autonomous devices called sensors or nodes that are cooperatively sensing, computing and wirelessly communicating with each other. Nodes in WSN are severely constrained in their power, memory and computations. The battery powered nature of sensors vulnerable the sensor life time and hence the WSN life time. Size of the network can be flexibly change by adding or removing nodes and this will unpredictably change the topological construction of the network. The main challenges in WSN are battery capacity, bandwidth and computing power. In order to extend network life time we need to preserve the amount of power and to conserve the network energy [1]. Hence, routing and clustering algorithms applied to provide long-range and large-scale WSNs communications. Routing in WSN is differ conventional routing in fixed networks. Selecting the shortest path between source and sink is not always mean optimal routing in WSNs. The scarce power in sensors challenges the routing protocol in WSNs. Therefore a power awareness based routing algorithms should be introduced to preserve WSN power and hence extend the network life time. Because of WSNs constrains and limitations, design of routing protocols for WSNs is challenging. 
Classical WSN routing protocols are categorized to three main categories: flat-based routing, hierarchical-based routing and location-based routing depending on the network structure. To overcome WSN challenges, the intelligence and flexibility of soft computing paradigms in processing the ambiguity and uncertainty of the data in complex environment has attract researches`attentions to the idea of employing embedded soft computing methods in WSN after deployment. The characteristics of soft computing show great analogy and compatibility in wireless sensor networks especially in power management approaches, self-decision making techniques; knowledge-based routing and nodes processing [2].

In this paper we survey the proposed routing protocols in WSNs based on Soft Computing paradigms. Firstly we will outline the characteristics, design objectives and challenges in WSNs. This will secondly followed by a comprehensive survey of the different categories of WSNs routing protocols. We will thirdly introduce, analyse and sort the usability of different Soft Computing paradigms in routing WSNs. The conclusion of the paper provides a good insight to future research areas in routing WSN based on Soft Computing paradigms.

\section{WSN CharaCteristics, Design ObJectives, And Challenges}

In area of WSN, applications vary and depend on a wide range of technologies, characteristics, design objectives and challenges of WSN. Therefore application requirements on WSNs differ from one application to another. A few explorations that should be taken in consideration is mentioned and discussed below.

\subsection{Characteristics}

As wireless sensor network (WSN) is recently considered as one of the most important telecommunication technologies that proves its compatibility and reliability in many applications disciplines. Based on references [3, 4] WSNs uniquely have the following distinctive characteristics:

- Dense self-deployment: WSN is a huge distributed computational system. Large number of sensors are scattered and densely randomly deployed in the network environment. Sensors are configured autonomously as each sensor independently manages its selfcommunication in the network.

- Limited processing and storage: Sensor nodes are small battery powered autonomous physical devices that highly limited in, computational capabilities and storage capacity.

- Limited energy resources: Due to the tough nature of WSN applications environment and the fact that sensor nodes are battery powered devices, it is usually hard to change or recharge theses batteries.

- Sensor heterogeneity: Since sensor nodes existence is not guaranteed in the WSN life time, unreliable and inconsistent sensor nodes will prone due to physical damages or failures while harsh deployment.

- Data redundancy: Data can be sent differently by more than one node to central node due to the need of collaboration and communication of sensor nodes as well as the physical nature of the sensor nodes.

- Application centric: As it is always hard to change or modify in the wireless sensor network, the network is usually designed and deployed for a specific application. This mainly affects the design requirements, network size, energy consumption and routing constrains of network.

- Broadcast communication: Sensors in WSN usually depend on exchanging sensed data between multiple sensor nodes and particular sink node using different flooding routing techniques. 
- Topological inconstancy: Due to power scarcity in sensor nodes as well as the harsh environment, Network topology will usually suffer frequent changes such as connection failures, node death, adding new node, energy consumption or channel fading.

- Limited transmission range: The limited physical characteristic of sensor nodes are usually limited strictly the network capabilities and affect the coverage range and communication quality.

\subsection{Design objectives}

The design of WSN generally depends on the applied area of application as each application has its different requirements, however some general design objectives should be taken in consideration while deploying WSN $[3,4,5]$. The following are the most common design objectives:

- Network size, cost, resources: WSN size mainly depends on the size and coverage of geographical area of the deployed network for a specific application. The number of sensor nodes varies to thousands and even more. Size of WSN mainly affects the required nodes number, cost, routing techniques and connection technology. This also will directly affect the network scalability and feasibility.

- Network topology: One of the main aspects in the WSN design that affects network capacity, complexity, delay and routing. The size of the network and the area of interest determine the network topology. WSN topology is a dynamic topology that may be simple with single or few numbers in direct communication hops between the nodes or complex with multi-hop complex topological architecture.

- Power consumption: The physical nature of the sensor nodes constrained it with very limited energy resources. Sensors mainly depend on batteries as power suppliers. According to the harsh environment for WSN, it's almost hard or even impossible to change or replace these batteries. The overall network life time is a cumulative of its sensors life. Therefore, preserving network life urges researches to focus on the development of an efficient power management approaches and routing protocols that manage and control the consumption of sensors`energy.

- Coverage range: In order to preserve the network consumed energy and to increase its productivity and reliability, network coverage range should be selectively determined. Small transmission range between nodes will decrease the amount of needed power for transmission between directly connected nodes. The huge coverage areas usually cased an eavesdropping.

- Quality of service: The area of WSN application restrains the provided quality of service in WSN. For real time applications, sensed data should be delivered as soon as it is sensed. The frequent changes in the sensed data are highly effected with the time factor. Reliability and usability usually depend on QoS.

- Simplicity: The heterogeneous and autonomous nature of sensors in WSN as well as the complex topological nature requires simple and convenient communication, processing and power consumption models in order to ease and increase the efficient utilization of the network.

- Mobility: The mobility nature in WSN after deployment usually results due to the automotive capabilities of sensor nodes. Each sensor has the flexibility to change its location based on some environmental factors that strongly affect nodes movements are varies based on the application area. Mobility may apply to all nodes within a network or only to subsets of nodes. Therefore based on the mobility taxonomy, sensors in WSN expand to different status. They may act as an active or passive nodes, this is addressed by each sensor's automotive capabilities, so the sensor may depend in motion device or move by itself. The motion as well may be occasional movement with time intervals of 
immobility in between, to constant as in static nodes. Although the high degree of mobility in WSN, some sensors may remain static. The extent of dynamic in WSN as well as the speed of mobility frequently influences the size, design and protocols of the network.

- Fault tolerance: The ability to preserve the network performance and functionality even after individual node failure or congestion in some of parts of the network. The adaptability of WSN can be achieved by using efficient routing protocols, power management approaches and communication establishments.

\subsection{Challenges}

Deployment of WSN face a number of great challenges that urge researches to focus on it in order to reach an optimal performance of WSN, below are some general challenges that have been faced in different WSNs applications $[6,7,8]$.

- Hardware constraint: Since WSNs depend on battery based power devices; power supplier is the most important part in the sensor nodes. The less energy consumption devices in WSN are the most efficient and lasting WSN. The characteristic of sensor nodes; such as the computational capabilities and storage capacity; will also affect the performance and life time of WSN as they may increase the energy consumption and data redundancy. The size, processing, cost and the amount of the sensors in the applied environment should be taken in consideration while we develop WSNs.

- Power consumption: The limitation of power resources in WSNs vice versa the high energy consumption direct the researchers attentions to power conservation and power management approaches that will effectively prolong the WSN lifetime.

- Deployment: A WSN is an infrastructure less randomly deployed network consist of small autonomously distributed sensors. The network deployment can be densely by a huge number of sensors in applied area of application or sparse network with a few and limited number of sensors. Communication in WSNs is achieved by single or multi number of hops between sensors. The importance of application as well as the cost of deployment controls the class of WSN deployment.

- Scalability: WSNs should be able to support variety of routing protocols, huge nodes number and wide area of application as well as the frequent increases of network expansion. The scale of performance and workload of WSN should not be anticipated during the initial network design stage.

- Flexibility: Due to the wide diverse of WSN application, as well as the network constraints and scarcity of resources, some sort of flexibility are needed such as different network deployment schemes and topologies, routing protocols, power management methods and so on.

- Reliability: A WSN should be able to adapt and manage the corruption of the network in case of node failure. The functionality and performance to WSNs should not be affected negatively. Some fault tolerance techniques ensure reliability in WSNs.

- Connectivity: Maintain connectivity among all sensor nodes through the network life time is a very challenging issue. The importance of each sensor node as well as the importance of sensed data and routing route that each sensor may take urges the network to preserve the life of each node. Some sleep modes can be practiced by some nodes in order to reduce the rate of harvested energy.

- Lifetime: The longevity and coverage of the WSN should be guaranteed. The main emphasis is to prolong the network lifetime. Sensor nodes are finite life time devices as they are battery powered. Some adapting mechanisms such as power management techniques and adaptive routing protocols are used to overcome the limited resources efficiently and to ensure the maximum network lifetime. 


\section{WSN Routing Protocols}

Due to the secrecy of energy resources in WSN, prolonging the network life time is considered as a challenge problem. Although network life time is affected by the limitations of the battery powered devices, the length of travelling path as well as balancing the load on a specific path and the reliability of this path will also greatly affect the life time of WSN. Data in WSN travels through its route from the source node to another neighbor selected successor node. It repeatedly does this movement based on specific selection methods until reaching the sink node. Routing in WSN can be classified either depending on network structure or protocol operation. Below are a few explanations for the routing protocols [9].

\subsection{Network Structured Based Routing}

In this section we survey the state-of-the-art routing protocols for WSNs. In general, routing in WSNs can be classified flat-based routing where every node play the same role in the network, hierarchical-based routing where the nodes play different roles and location-based routing where data are routed according to nodes position depending on the network structure [9].

- Flat-based routing: In this routing protocol, equal roles and functionalities are assigned to all nodes. All nodes have the same roles on the network while they don't follow any structural distribution. Nodes communicate directly and indirectly with the base station. Base stations play main role in order to communicate with the huge number of nodes. Each node senses nearest neighbor nodes and transfer data based on data centric routing mode. The main advantage of flat routing is the simplicity as there are no overheads. It also guarantees scalability due to the fact that nodes have the same role and functionalities. The network lifetime is maximized time by taking the benefit of multi hops routing that balanced the carried load by restricting the power level at which sensor nodes communicate. However the main disadvantage of this routing protocol is that fairness among nodes is not guaranteed and this may lead to the concept of hotspot when nodes are uniformly distributed in WSN. So if there is only one sink node in WSN, the energy of the nodes that surrounds the sink will be highly consumed and this may decay the network lifetime [10]. Flooding, Gossiping, Sensor Protocols for Information via Negotiation (SPIN) and Directed Diffusion (DD) are flat routing protocols. Flooding depends on sending the data to all the nodes in the network, so each node receives the data will resend it again to all its neighbors. Gossiping based on flooding but only one randomly neighbor will be selected to forward the data and this will greatly reduce the consumed energy. SPIN and directed diffusion are mainly eliminating the redundant data and use data negotiation to conserve WSN power.

- Hierarchical-based routing: In this routing protocol, different roles, capabilities and functionalities are assigned to all nodes. All Nodes are playing different roles on the network. Network is divided to a number of clusters. Each cluster consists of number of nodes and nominates only one node to be the head of the cluster. Messages on the network are sent from nodes only to the cluster head and hence cluster head forward this message to the sink. Cluster head responsible for manage, collect, aggregate and retransmit data from cluster nodes to base station. Cluster preservation depends on intelligence of detection and recovery determining the survival of cluster head. The cluster head selected and assigned periodically in order to eliminate the overall consumed energy of the network and to prolong the network life time. The tackled problem of this protocol is massive power consumption in cluster head. Therefore it is always recommended to periodically rotating the cluster heads among the nodes to ensure uniform energy consumption and to prevent energy hotspot. Energy conservation in clustering greatly contributes to overall system scalability, lifetime and energy efficiency 
[11]. The main advantage of hierarchical-based routing is the data aggregation as the data of the node in the cluster can be combined with the cluster head and this will reduce the data redundancy. However hierarchical-based routing has many disadvantages such as being a hotspot as a result of cluster head election, the need of excessive energy of cluster head, the deployment complexity in order to balance power consumption and the lack of scalability that increase the messages overhead in clusters. Some examples of hierarchical-based routing are Low-energy adaptive clustering hierarchy (LEACH), Threshold sensitive Energy Efficient sensor Network protocol (TEEN), Adaptive Threshold sensitive Energy Efficient sensor Network protocol (APTEEN), Powerefficient Gathering in Sensor Information Systems (PEGASIS) and Minimum energy consumption network (MECN). LEACH forms clusters of the sensor nodes based on the received signal strength and use the local cluster heads as gateway to the base station. TEEN is a hierarchical protocol designed to be responsive to sudden and drastic changes in the sensed attributes such as temperature, pressure, rainfall, etc. APTEEN aims at both capturing periodic data collections and reacting to time critical events. PEGASIS forms chains of sensor nodes so that each node transmits and receives from a neighbor and only one node is selected from that chain to transmit to the base station rather than forming multiple clusters. MECN finds a sub network of the WSN with less number of nodes and finds the minimum global energy required for data transfer.

- Location-based routing: In this routing protocol, the position of sensor nodes' exploit to route data in the network. All nodes in the network are addressed by their locations. Location information used to investigate, select and maintain the optimal route to forward the data packets. It based on the frequent calculations of distances between nodes and the estimation of consumed energy level. This depends on the frequent updates of the nodes' location information. Power management approaches are used to reduce energy consumption and prolong network life time by setting some nodes into sleep mode in its deactivation status. Routing protocols mainly depend on area partitioning schemes and location information. The advantage of using location information-based routing algorithm is the ease and optimization to manage the network as well as reducing the control overhead of the network [12]. However the main disadvantage of location-based routing is the complexity in designing the sleep mode of nodes. Examples of Locationbased routing are Geographical and Energy Aware Routing (GEAR) and Greedy Perimeter Stateless Routing (GPSR). GEAR mainly focus on optimize power consumption by using energy aware and neighbors location information to set the forwarding route. GPSR use the perimeter of the planar graph to find the optimal route for sending the packets. This requires a location service to map locations and node identifiers.

\subsection{Protocol Operation Based Routing}

These routing protocols are mainly based on the protocol operation. Different routing functionality can be applied according to the variation of the approach used in the protocol. Summary below the taxonomy of different protocol operation based routing which is deeply discussed in [12]:

- Negotiation-based routing: This routing protocol is based on exchanging a number of negotiation messages between interconnected nodes. The advantage is that it works to reduce data redundancy and prevent information duplicate [13]. Examples of negotiationbased routing are Sensor Protocols for Information via Negotiation (SPIN) that uses negotiations to address all problems of flooding as it uses meta-data to succinctly and completely describe sensor data. Sequential assignment routing (SAR) that creates multiple trees, each rooted at a 1-hop neighbor of the sink, to establish multiple paths 
from each node to the sink. Directed Diffusion (DD)uses flooding based query mechanism for continuous and aggregate queries.

- Multipath-based routing: This routing protocol is based on finding other possible better paths between sources and sinks to increase routing efficiency and reduce power consumption in WSN. The advantage of this protocol lies on reserving the level of consumed power and thus prolog the network life time [14, 15]. It also helps with fault tolerance and quick recovery from broken routes. The network performance will efficiently increase by reducing the transmission delay as well as the network reliability will be guaranteed due to overheads. However the disadvantage of it is the great amount of overheads and energy consumption as a result of sending periodic messages to keep the network paths alive. Establishing and maintaining all trees is expensive. Examples of this protocol are Multi path and Multi SPEED (MMSPEED) that provides QoS differentiation in terms of timeliness and reliability, while also minimizing protocol overhead. Sensor Protocols for Information via Negotiation (SPIN) that uses negotiations to address all problems of flooding as it uses meta-data to succinctly and completely describe sensor data.

- Query-based routing: This routing protocol is based on a series of propagated queries between the sources and sink node to sense the travelling paths. The destination node sends query of interest from a node through network and node with this interest matches the query and send back to the node which initiated the query. The query normally uses high level languages. The efficient route discovered and constructed by the updated information related to each route in the network nodes [16]. The advantage of this routing protocol is that it eliminates redundancy and reduces number of transmissions across the network. The disadvantage of query-based approach is that it may not be best solutions for networks with need for continuous data transfers such as environmental monitoring. Examples of Query-based routing are Sensor Protocols for Information via Negotiation (SPIN) that uses negotiations to address all problems of flooding as it uses meta-data to succinctly and completely describe sensor data and Directed Diffusion (DD) uses flooding based query mechanism for continuous and aggregate queries.

- QoS-based routing: This routing protocol is based on balancing between all the network constraints to satisfy all the QoS metrics such as energy consumption, data quality, delay, priority level and bandwidth and so on [17]. Disadvantages of this protocol mainly lie on the delay to meet the QoS metrics. This will consume the network energy massively. As well that it is expensive to establishing and maintaining all trees on the network. Examples of QoS-based routing are Sequential assignment routing (SAR) that creates multiple trees, each rooted at a 1-hop neighbor of the sink, to establish multiple paths from each node to the sink. This will minimize the average weighted QoS metric over the lifetime of the network. Multi path and Multi SPEED (MMSPEED) that provides QoS differentiation in terms of timeliness and reliability, while also minimizing protocol overhead.

- Coherent-based routing: This routing protocol is based on the two data processing techniques in WSN, coherent and non- coherent. Energy efficient route will be selected based on the amount of processing. Coherent routing forwards data after minimum processing to eventually reduce the consumed energy. While non-coherent routing send data after perform local processing in each node [17]. The advantage of coherent data processing routing is the energy efficiency due to the preprocessing on data and data aggregation. While the non-coherent processing involves target detection, as data collection and preprocessing of its data takes place. Then neighbors cooperation where neighboring nodes must be aware of the local network topology. Finally select central node to refine information processing. Disadvantage is that central node must have enough energy resources and computation abilities. 


\section{Soft Computing Paradigms For WSN Routing}

The efficient utilization of energy in WSN is a bottleneck problem that affects the performance and the life time of network $[18,19]$. Energy consumption attentiveness and power management approaches are recently addressed by researches to tackle this problem. Optimal routing technique and energy optimization usage are significantly affecting the WSNs performance and guarantee the extension of the network life time. Due to WSNs constraints and especially the sensors`energy scarcity, a smart routing should be done to balance the energy consumption among nodes, therefore prolonging the network lifetime and insuring network coverage. Deploying smart and intellectual techniques enhances the effectiveness of wireless sensor network [20].Different soft computing paradigms have been studied and examined by researches to optimize WSN routing with the consideration of the power consumption, network challenges and design and deployment aspects. The soft computing paradigms such as Reinforcement Learning (RL), Swarm Intelligence (SI), Evolutionary Algorithms (EA), Fuzzy Logic (FL), Neural Networks (NN) and Artificial Immune System (AIS) have been applied to different WSN applications and deployment based on their different characteristics. The section below in this survey paper summarizes the recent implementations of soft computing paradigms in routing in WSN with its dynamical and heterogeneous characteristic. Different surveyed soft computing paradigms based on their efficiency, suitability and usability in WSN are listed below. The presentation of the mentioned soft computing paradigms below are sorted and organized based on their precedence usability and efficiency in WSN routing. This paper will serve as a guide for using SC paradigms for WSNs.

\subsection{Reinforcement Learning (RL)}

This soft computing paradigm works on maximizes the system performance by using simple reward feedback for the agent to learn its behavior. It has the largest number of publications on our research area. Reinforcement learning (RL) proves to be the most appropriate and usable soft computing paradigm in WSN routing. Position of sensor nodes in the network and the distance from sink node greatly affect the level of energy consumption in each node. Each node needs an adaptive energy consumption and optimization algorithm to improve performance and preserve the energy of the network. The idea behind the usability of RL lies specifically in the Q-learning algorithm that is able to construct better solutions for distributing and dynamic problems such as clustering and routing approach with the minimal communication and computational requirements [20]. The comprehensive contextual paradigm of RL provides a perfect model that matches with the nature of WSNs. Having a reinforcement learning algorithm will support the nodes to maximize the power level and minimize the distributed system latency.

Paper [21]; Boyan and Littman are the pioneers who used Q-learning algorithms [22, 23] in WSNs routing techniques. Their paper described the Q-routing algorithm for packet routing, in which a reinforcement learning module is embedded into each node of a switching network. Only local communication is used by each node to keep accurate statistics on which routing decisions lead to minimal delivery times. Q-routing proves superior to a non-adaptive algorithm based on pre-computed shortest paths and is able to route efficiently even when critical aspects of the simulation, such as the network load, are allowed to vary dynamically.

Paper [24] studied the potential of using energy aware metrics in reinforcement learning based routing algorithms for wireless sensor networks. It contributed with an enhanced version of an 
existing energy aware algorithm and with a study that tests the influence of combining energy aware metrics with load balancing metrics from delay based Q-routing. Results showed that the enhanced algorithm can significantly improve the lifetime of a network without requiring any extra information or communication, by propagating energy information beyond direct neighbors throughout the network. It showed that topologies composed from heterogeneous nodes can have a significant impact on an algorithm's performance. Furthermore it explored that load balancing in routing algorithms can help to improve the network lifetime while only requiring energy information about a node's direct neighbors.

Paper [25] used a reinforcement learning algorithm with the aim to increase the autonomous lifetime of a Wireless Sensor Networks and decrease latency in a decentralized manner. The main challenges in WSNs are the limited power supply of nodes and the need for decentralized control. To overcome these challenges, authors made each sensor node adopted an algorithm to optimize the efficiency of a small group of surrounding nodes, so that in the end the performance of the whole system was improved. They compared their approach to conventional ad-hoc networks of different sizes and show that nodes in WSNs are able to develop an energy saving behavior on their own and significantly reduce network latency, when using their reinforcement learning algorithm.

Paper [26] described how information local to each node can be shared without overhead as feedback to neighboring nodes, enabling efficient routing to multiple sinks. Such a situation arises in WSNs with multiple, possibly mobile users collecting data from a monitored area. Authors formulated the problem as a reinforcement learning task, and applied Q-Routing techniques to derive a solution. Evaluation of the resulting protocol demonstrated its ability to significantly decrease the network overhead over existing approaches.

Paper [27] proposed new modality for sensor networks to emerge when considering multiple, distributed base stations that collect data from sensors. This scenario reversed the typical multiple-source, single-sink scenario, and required new techniques to efficiently send data from single-sources to multiple-sinks. While an offline approach with full topology information can build the optimal data forwarding tree, the challenge that the paper addressed here was to optimize data forwarding with only information exchanged among one-hop neighbors. The novelty of this approach lied in the use of an iterative learning technique that explored alternative routes by locally sharing feedback regarding route fitness. This paper presented the approach as well as an evaluation that showed that the learned paths eventually increased the network lifetime of up to $50 \%$ over an approach without learning.

\subsection{Swarm Intelligence (SI)}

This soft computing paradigm is an evolved system of collective intelligent groups of simple agents that interacts with each other's and the environment around. It is characterized with decentralization. Individual agents act by following simple rules that accumulatively lead to global system behavior. SI is the second powerful soft computing paradigm that proves a great compatibility in WSNs routing. Due to the environmental ontological match between WSN and SI, efficient routing techniques can be achieved. SI addresses the management of collective behaviors of highly dynamic and distributed elements in decentralized and self-deployed systems.

The novel idea of SI is to optimize the distribution of uncontrolled systems [28, 29, and 30], therefore, based on such a state-of-the-art some routing techniques can be introduced to find shortest path in ants` colony [31]. Researchers invented a number of techniques such as AntBased routing protocols; Basic Ant-Based Routing (BABR) Algorithm, Sensor-driven and Costaware ant routing (SC), Flooded Forward ant routing (FF), Flooded Piggybacked ant routing (FP), 
and Energy-Efficient Ant-Based Routing (EEABR) [32]. The main idea behind all these techniques is to find the optimal shortest path between sender and receiver nodes based on energy aware methods to maximize the network lifetime [32]. Propagation from one node to another along the transmission path depends on the calculation of the neighbor nodes energy function and the amount of pheromone trail present on the connections between the nodes. After the data delivery, a backward journey take place on same constructed path to update with number of nodes and amount of consumed energy. Routing hierarchy with optimized energy branches constructed after number of trials [31].

Paper [33] examined the swarm intelligence phenomena in three different routing algorithms in WSN. The algorithms SC, FF and FP successfully proved a good system start-up with some latency, while providing better energy efficiency. Besides, the FP algorithm, while providing high success rates of data delivery, consumed much higher energy than the FC and FF algorithms. The performance evaluations for these algorithms on a real application were conducted on a routing simulator for sensor networks and proved good results in WSN routing.

Paper [34] proposed an Ant Colony Optimization meta-heuristic algorithm for routing in WSNs. The protocol was studied by simulation for several Wireless Sensor Network scenarios and the results clearly showed that it minimized communication load and maximized energy savings.

Paper [35] proposed a particle swarm optimization based routing (PSOR) algorithm that counted on nodes energy level as well as the lengths of the routed paths to maintain network life time at a maximum. A comprehensive comparison between the proposed model and genetic algorithm has been practiced on different sized networks. Furthermore instead of forming greedy chain or cluster of chain, which may not always ensure minimum energy dissipation, authors used an intelligent particle swarm based optimization particle swam optimization is an inspiration from the flocking birds.

While SI routing proved great flexibility in routing WSN, it still suffers the problem of generating too much additional traffic. The forward and backward ants that move through the network may not provide a very flexible routing scheme, but a lower overhead.

\subsection{Evolutionary Algorithm (EA)}

This soft computing paradigm is an artificial intelligence computational optimization algorithm for population heuristic. Genetic algorithm (GA) is one of the most popular EA algorithms that mimic the process of natural evolution. EA hasn`t approve a great optimality in WSN routing. As maximizing the lifetime period in WSN depends on energy consumption of the node, selecting optimized path between source and the sink is key challenge. The calculation of cost function of path between source and the sink must be calculated in order to find the shortest path. Greatness of evolutionary algorithms lies on genetic algorithm that solves unconstrained problems and able to find the optimized path between nodes based on constrained function [36-38].

Paper [39] focused on minimizing the consumed energy of the network based on the heuristics of GA. The cost function of selection depends on the link quality residual energy and number of successive transmission. The occurrence of any event in the network urged all the nodes in the range to sense and gather information. These gathered information then delivered to the upstream head node. The proposed routing algorithm took the next hop selection based on the minimum cost function value. Genetic algorithm used to optimize the minimum cost function. Using evolutionary optimization method, minimum number of nodes was selected to obtain the optimal route. 
Papers [40, 41] also applied a very contextual experiment using GA in optimizing routing in WSNs based on finding the shortest path. Genetic algorithm with elitism concept was used to obtain energy efficient routing by minimizing the path length and thus maximizing the life of the network. Inheritance of GA will greatly preserve the elite solutions to the next generations in order to converge to the global optima of finding the shortest route. Results showed a great efficiency and optimality of GA in preserving power consumption and converging to the optimal route.

Paper [42] considered the synthesis of a 2-D network of $\mathrm{N}$ transceiver nodes with a known spatial distribution using a genetic algorithm optimization method. Each of the nodes consists of a relatively simple a transceiver (antennas, a receiver and a transmitter) in which only limited adjustments of antenna pattern and transmission power level are available. The goal of the optimization was to maximize nodal signal to noise ratios (SNR) while minimizing the transmitter power levels.

Paper [43] proposed a genetic algorithm for designing a wireless sensor network based on complex network theory. It developed a heuristic approach based on genetic algorithms for finding a network configuration such that its communication structure presents complex network characteristics. The mathematical model of the hub location problem was developed to determine the nodes which will be configured as hubs. This model was adopted within the genetic algorithm. The results revealed that this methodology allowed the configuration of networks with more than a hundred nodes with complex network characteristics, thus reducing the energy consumption and the data transmission delay.

Paper [44] proposed the same ideas of an efficient method based on genetic algorithms (GAs) to solve a sensor network optimization problem. However what make this paper unique is that its approach is also applicable to multiple network topologies such as uniform, non-uniform or shortest distance optimization problem.

\subsection{Fuzzy Logic (FL)}

This soft computing paradigm is a flexible mathematical and computational model that deals with fuzziness and uncertainty of data. As WSN is an uncertain environment with some insufficient data, it needs special decision making. It needs some flexible and tunable procedures that deploy routing and enhance the overall network lifetime [45]. Using fuzzy logic will avoid WSN the complex mathematical modeling as well as provide it with great flexibility to deal with uncertainty and imprecision along the network lifetime. The critical tradeoffs in WSN such as minimized consumed energy vs. transmission route, multi-hop vs. direct communication, computation vs. communication [46].

Paper [47] solved the problem of the growing demand for real time services in wireless Sensor Networks (WSNs); Quality of Service (QoS) based routing. Fuzzy Logic System (FLS) is used as a decision mechanism for next hop node selection. Both transmission rate and energy were chosen parameters for choosing the next-hop node in real-time packet transmission. Simulation results showed that the proposed scheme provided improvement on real-time transmission and energy efficiency performance, when operating in varying real-time environment.

Paper [48] explored the optimization of energy usage in wireless sensor networks. As routing is one of these areas in which attempts for efficient utilization of energy have been made, these attempts use fixed (crisp) metrics for making energy-aware routing decisions. The paper, presented a generalized fuzzy logic based approach for energy-aware routing in wireless sensor networks. This generalized approach is soft and tunable and hence it can accommodate sensor 
networks comprising of different types of sensor nodes having different energy metrics. The simulation results have demonstrated the reliability and efficiency of this approach.

Paper [49] improved LEACH routing protocol using Fuzzy Logic (LEACH-FL), which takes battery level, distance and node density into consideration. The proposed method has been proved making a better selection.

Paper [50] proposed novel energy efficient algorithm FDPCA for Wireless Sensor Networks (WSN). Parameters like End to End Delay and Received Signal Strength Indicator (RSSI) are considered for exercising the influence on transmit power. These parameters are fuzzified and optimal transmission power levels are selected. The throughput for both DPCA and FDPCA are compared. High throughput is obtained by using FDPCA. In first phase, the parameters are calculated. The proposed algorithm can effectively save energy without degrading the throughput of the network and reduce the energy consumption of the network. The experimental results demonstrate that the proposed algorithm significantly overtake previous method, in terms of throughput.

\subsection{Neural Network (NN)}

This soft computing paradigm is a learnable arithmetical algorithm that maps complex relation between input and output based on supervised learning methods in different environments [51]. Applying the neural network paradigm in context of wireless sensor network provides deep understanding of WSN. The architectural match between neurons in ANN and sensor nodes in WSN as well as the connectivity paradigm presents a great analogy between WSN and ANN. Accordingly replacing traditional signal processing algorithms in WSN by simple computation ANN carries out efficient implementation as well as resource reduction in WSN [52].

ANN strongly proved its compatibility in WSN and effectively ensures its prolonged existence. It is able to predict and dimensionally reduce the amount of consumed energy in sensor nodes and hence the amount of required energy for each route [53]. While clustering significant to improve the network lifetime, we need to reduce the consumed energy and increase the scalability of the sensor network. Pre-assignment and election of cluster head can produce scalable sensor network while reducing the consumed energy can efficiently achieved of cluster based routing. Sensing nodes can be developed using ANN and reduce energy through some series of power measurements and prediction [54]. With the simplicity of NN, we can't strongly agree its compatibility in WSN routing. NN have some challenges that can't cope with the nature and the frequent changes WSN properties. It requires an offline learning phase and too many calculations that can`t easily adopt with the WSN topology.

Paper [55] optimized the routing in WSNs by using an appropriate path discovery model regardless of the $\mathrm{NN}$ constraints on data processing and power consumption. SOM used in each single node to manage and discover the optimal route to follow. This will greatly improve the WSN performance.

Paper [56] proposed minimum power intelligent routing model in WSNs using self-organizing neural networks. This built a power optimization and energy conservation methods that based on each node role, routing will be classified according to the node importance and due to their positions to reach the short optimized transmission route.

Paper [57] proposed a neural network solution for optimized power management of a wireless sensor network. A prediction of power level in sensors can be calculated based on defined set of attributes. This will help to manage the network tasks as well discovering the optimal routes to 
WSN. A set of attributes based on sensors' location and neighbourhood have been defined and used as inputs of neural network and the output of the neural network will be used as a factor in the route path discovery and power management. The proposed model observed that the neural network can predict the final power level of each sensor with approximately $13 \%$ of precision.

\subsection{Artificial Immune System (AIS)}

This soft computing paradigm is computational system that inspired from the biological human capabilities to imitate intelligent human behaviour in computerized tasks and problem solving. AIS hasn't prove any compatibility in routing WSN and it's rarely applied in WSN. Recently AIS shows that clustering and cluster head selection can be efficiently applied. Routing and clustering problem in WSN mainly depend on static and fixed number of clusters in the network. AIS can be a new research area that haven`t well studied yet. Few papers only found to prove its compatibility with clustering.

Paper [58] focused on the number of cluster heads and minimizing the distance of the overall transmission. The way that researcher minimize the total communication distance guarantees a great extension of the network life time.

Paper [59] used a very special way to prolong the network life time. It focuses mainly on decrease the level of consumed energy in each node. The experiment led to scalable routing algorithm by selecting the next routing nodes using AIS. AIS mainly used to cluster the WSN nodes into a number of independent clusters in order to minimize the routing distance.

\section{CONCLUSION AND FUTURE WORK}

Routing and clustering in wireless sensor networks are the most challenges of WSN. This paper presents a comprehensive survey of the most updates area of routing in WSNs based on soft computing paradigms. The innovative use of soft computing paradigms in WSNs energy conservation methods proves a good compatibility with some variations through the proposed SC paradigm. Table 1 summarizes the properties of SC paradigms in routing WSN.

Table 1. Properties of SC paradigms in routing WSN

\begin{tabular}{|l|l|l|l|l|}
\hline SC Paradigm & Computation & Memory & Flexibility & WSN Routing Optimization \\
\hline RL & Low & Medium & High & Most appropriate \\
\hline SI & Low & Medium & High & Moderately appropriate \\
\hline EA & Medium & High & Low & Less appropriate \\
\hline FL & Medium & Medium & High & Moderately appropriate \\
\hline NN & High & High & Low & Less appropriate \\
\hline AIS & Medium & Medium & High & Not appropriate \\
\hline
\end{tabular}

The studied SC paradigms prove that not all of them are equally compatible for routing in WSNs. It was clear the RL is the best paradigm for dynamic and distributed systems such as WSNs. It works optimally in routing and clustering problems in WSNs due to its flexibility and robustness. It doesn't require any overhead connections and preserve the data delivery under any topological changes. The SI paradigms show the need to be changed to accommodate the WSNs requirements and properties. It is usually requires a backward travel to the source, which increases the level of energy consumption. FL can be a good choice to solve the routing and clustering problems however it needs a frequent learning process for the fuzzy rules based on the 
frequent topological changes that may happen in WSNs. NN and EA require too much processing. However $\mathrm{NN}$ is a suitable paradigm for clustering and routing. It has a high processing demand and require high memory, therefore a pre deployed clustering schemes can be applied. To overcome the awkward of NN in WSNs routing, researchers should deeply focus to apply different models of NN in WSNs applications. Therefore it is challenging as a future work, to explore more the capabilities of NN in routing WSNs as it rarely applied. Detailed and comprehensive studies of $\mathrm{NN}$ can be modelled, investigated and examined for different WSNs platforms and environments. We will mainly try to focus on optimizing WSNs routing based on NN. Therefore an efficient WSNs routing protocol will be selected and optimized by NN. The flexibility of NN will promisingly achieve a great compatibility in routing WSNs but a general evaluation remains open to future research. The hybrid Bio-inspired computing paradigms are also promising for solving optimization in WSNs.

\section{REFERENCES}

[1] I F Akyildiz, W Su, Y Sankarasubramaniam and E Cayirci (2002) "Wireless sensor networks: a survey”, Computer Networks Vol. 38, No. 4, pp. 393 - 422.

[2] Averkin, A. N., Belenki, A. G., \& Zubkov, G. (2007) "Soft Computing in Wireless Sensors Networks", In Conference of European Society for Fuzzy Logic and Technology (EUSFLAT), No.1, pp. 387-390.

[3] Zheng, J., \& Jamalipour, A. (2009)"Wireless sensor networks: a networking perspective", published by A John \& Sons, Inc., Wiley. com

[4] Romer, K., \& Mattern, F, (2004)"The design space of wireless sensor networks", Wireless Communications, IEEE, Vol. 11, No. 6, pp. 54-61.

[5] Yoneki, E., \& Bacon, J. (2005)"A survey of Wireless Sensor Network technologies: research trends and middleware's role", University of Cambridge TR, 646.

[6] Seah, W. K., Eu, Z. A., \& Tan, H. P. (2009)"Wireless sensor networks powered by ambient energy harvesting (WSN-HEAP)-Survey and challenges", Proceedings of the 1st International Conference on Wireless Communications, Vehicular Technology, Information Theory and Aerospace \& Electronic Systems Technology (Wireless VITAE), pp.1-5.

[7] Kulkarni, R. V., Forster, A., \& Venayagamoorthy, G. K. (2011)"Computational intelligence in wireless sensor networks: A survey", Communications Surveys \& Tutorials, IEEE, Vol. 13, No. 1, pp. $68-96$.

[8] A.Alkhatib and G.Baicher, (2012)"An Overview of Wireless Sensor Networks", 2012 International Conference on Computer Networks and Communication Systems (CNCS 2012) IPCSIT, Vol. 35.

[9] Park, J., \& Sahni, S. (2005)"Maximum Lifetime Routing In Wireless Sensor Networks" Computer \& Information Science \& Engineering, University of Florida.

[10] Datta, N. N., \& Gopinath, K. (2006)"A survey of routing algorithms for wireless sensor networks", Journal of the Indian Institute of Science, Vol. 86, pp. 569-598.

[11] Singh, S. K., Singh, M. P., \& Singh, D. K. (2010)”A survey of Energy-Efficient Hierarchical Clusterbased Routing in Wireless Sensor Networks", International Journal of Advanced Networking and Application (IJANA), Vol. 02, No. 02, pp. 570-580.

[12] Al-Karaki, Jamal N., and Ahmed E. Kamal (2004)"Routing techniques in wireless sensor networks: a survey", IEEE Wireless Communications, Vol.11, No. 6, pp. 6-28.

[13] Kulik, J., Heinzelman, W., \& Balakrishnan, H. (2002)”Negotiation-based protocols for disseminating information in wireless sensor networks", Wireless Networks, Vol. 8, pp. 169-185.

[14] Chang, J. H., \& Tassiulas, L. (2004)"Maximum lifetime routing in wireless sensor networks", IEEE/ACM Transactions on Networking (TON) Vol. 12, No. 4, pp. 609-619.

[15] Li, Q., Aslam, J., \& Rus, D. (2001)"Hierarchical Power-aware Routing in Sensor Networks", in Proceedings of the DIMACS Workshop on Pervasive Networking.

[16] Braginsky, D., \& Estrin, D. (2002) "Rumour routing algorithm for sensor networks", in Proceedings of the First Workshop on Sensor Networks and Applications (WSNA), pp. 22-31.

[17] Sohrabi, K., Gao, J., Ailawadhi, V., \& Pottie, G. J. (2000)"Protocols for self-organization of a wireless sensor network", IEEE Personal Communications, Vol. 7, No. 5, pp. 16-27.

[18] Pottie, G. J., \& Kaiser, W. J. (2000)"Wireless Integrated Network Sensors”, Communication of ACM, Vol. 43, No. 5, pp. 51-58. 
[19] Raghunathan, V., Schurgers, C., Park, S., \& Srivastava, M. B. (2002)"Energy-aware Wireless Micro sensor Networks", IEEE Signal Processing Magazine, Vol. 19, No.2, pp. 40-50.

[20] Forster, A. (2007)" Machine learning techniques applied to wireless ad-hoc networks: Guide and survey", The 3rd International Conference on Intelligent Sensors, Sensor Networks and Information Processing (ISSNIP), pp. 365-370.

[21] Boyan, J. A., \& Littman, M. L. (1994)"Packet routing in dynamically changing networks: A reinforcement learning approach", In Advances in Neural Information Processing Systems, No. 6, pp.671- 678 .

[22] Sutton, R. S., \& Barto, A. G. (1998)"Reinforcement learning: An introduction", MIT Press, Cambridge, Vol. 1, No. 1.

[23] Watkins, C. J., \& Dayan, P. (1992)"Q-learning”, Machine learning, Vol.8, No.3-4, pp. 279-292.

[24] Devillé, M., Le Borgne, Y. A., \& Nowé, A. (2011)'Reinforcement learning for energy efficient routing in wireless sensor networks", 23rd Benelux Conference on Artificial Intelligence (BNAIC 2011), pp. 89-96.

[25] Mihaylov, M., Tuyls, K., \& Nowé, A. (2010) "Decentralized Learning in Wireless Sensor Networks", Adaptive and Learning Agents, pp. 60-73.

[26] Forstert, A., \& Murphy, A. L. (2007) "FROMS: Feedback routing for optimizing multiple sinks in WSN with reinforcement learning", 3rd International Conference on Intelligent Sensors, Sensor Networks and Information Processing (ISSNIP), pp. 371-376.

[27] Egorova-Förster, A., \& Murphy, A. L. (2007) "A Feedback Enhanced Learning Approach for Routing in WSN", In Communication in Distributed Systems (KiVS), 2007 ITG-GI Conference, pp. 1-12.

[28] Okdem, S., \& Karaboga, D. (2009) "Routing in wireless sensor networks using an ant colony optimization (ACO) router chip", Sensors, Vol. 9, No. 2, pp. 909-92.

[29] Saleem, M., Di Caro, G. A., \& Farooq, M. (2011) "Swarm intelligence based routing protocol for wireless sensor networks: Survey and future directions", Information Sciences, Vol. 181, No. 20, 15, pp. 4597-4624.

[30] Murtala Zungeru, A., Ang, L. M., \& Phooi Seng, K. (2012) "Performance evaluation of ant-based routing protocols for wireless sensor networks", International Journal of Computer Science Issues (IJCSI), Vol. 9, Issue. 3, No. 2, pp. 388-397.

[31] Sarangi, S., \& Thankchan, B. (2012) "A Novel Routing Algorithm for Wireless Sensor Network Using Particle Swarm Optimization”, IOSR Journal of Computer Engineering (IOSRJCE), Vol. 4, Issue 1, pp. 26-30.

[32] Li, K., Torres, C. E., Thomas, K., Rossi, L. F., \& Shen, C. C. (2011) "Slime mold inspired routing protocols for wireless sensor networks", Swarm Intelligence, Vol. 5, pp. 183-223.

[33] Zhang, Y., Kuhn, L. D., \& Fromherz, M. P. (2004) "Improvements on Ant Routing for Sensor Networks", In Ant Colony Optimization and Swarm Intelligence, Springer-Verlag Berlin Heidelberg, Vol. 3172, pp. 154-165.

[34] Camilo, T., Carreto, C., Silva, J. S., \& Boavida, F. (2006)"An energy-efficient ant-based routing algorithm for wireless sensor networks", In Ant Colony Optimization and Swarm Intelligence, Springer Berlin Heidelberg, pp. 49-59.

[35] Sarangi, S., \& Thankchan, B. A (2012) "A Novel Routing Algorithm for Wireless Sensor Network Using Particle Swarm Optimization”, IOSR Journal of Computer Engineering (IOSRJCE), ISSN: 2278-0661 Vol. 4, Issue 1, pp. 26-30.

[36] Guo, L., \& Tang, Q. (2010) “An Improved Routing Protocol in WSN with Hybrid Genetic Algorithm", Second International Conference on Networks Security Wireless Communications and Trusted Computing (NSWCTC), Vol. 2, pp. 289 - 292.

[37] Kumar, M., Husian, M., Upreti, N., \& Gupta, D. (2010) "Genetic algorithm: Review and application", In International Journal of Information Technology and Knowledge Management, Vol. 2, issue 2, pp. 451-454.

[38] Li, X., Xu, L., Wang, H., Song, J., \& Yang, S. X. (2010) “A Differential Evolution-Based Routing Algorithm for Environmental Monitoring Wireless Sensor Networks”, Sensors 10, No.6, pp. 54255442.

[39] Nesa S., M.L Valarmathi, T.C. Neyandar (2011) "Optimizing Energy in WSN using Evolutionary Algorithm", International Conference on VLSI, Communication \& Instrumentation (ICVCI), International Journal of Computer Applications (IJCA) No.12. 


\section{AUTHORS}

Imane Aly Saroit received her B.Sc., M.Sc and the Ph.D from Faculty of engineering, Communication department, Cairo University. She is professor in Information Technology department and Vice dean for Education and Student Affairs, Faculty of Computers and Information, Cairo University. Her current researches focused on computer networks, specially wireless and mobile networks.

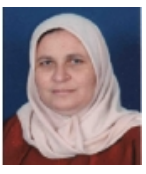

Hesham N. El Mahdy received his B.Sc. in Automobile engineering from the Military Technical Collage. He received his first M. Sc. in Computer Science from the Institute of Statistical Studies \& Research, Cairo University. He received his second M. Sc. in Computer Science from the University of Mississippi. He received his Ph. D. in Computer Science from the University of Mississippi. He is an Associate Professor in the Information Technology Department, Faculty of Computers and Information, Cairo University. His current research interests are Networks, Distance Learning, and Multimedia.

Eid Emary received his B.Sc., M.Sc and the Ph.D in information technology from Cairo University. . He is a lecturer in the Information Technology Department, Faculty of Computers and Information, Cairo University. His current research interests are Networks, image processing, and Multimedia.

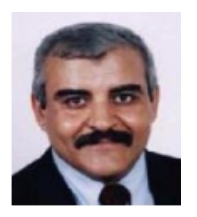

Marwa Sharawi received her B.Sc. and M. Sc in information technology from Cairo University. Currently she is a $\mathrm{PhD}$ candidate in information technology department, Cairo University. She is currently works as an IT lecturer in Arab Open University. Her current research interests are Networks and Artificial Intelligence.
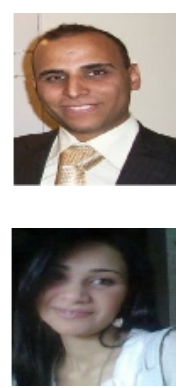